\title{
Comparison of plasma corticosterone concentration, muscle fibre diameter, and apoptotic markers between normal and pale, soft, exudative (PSE) turkey breast muscles")
}

\author{
MAGDALENA GÓRSKA, DOROTA WOJTYSIAK
}

\author{
Department of Animal Anatomy, Institute of Veterinary Sciences, \\ University of Agriculture in Krakow, Al. Mickiewicza 24/28, 30-059 Kraków, Poland
}

\section{Górska M., Wojtysiak D.}

Comparison of plasma corticosterone concentration, muscle fibre diameter, and apoptotic markers between normal and pale, soft, exudative (PSE) turkey breast muscles

\section{Summary}

The increasing production potential of birds is correlated with a lower health and immune potential, resulting in the appearance of numerous meat quality defects. The greatest concern for gallinaceous poultry (chickens and turkeys) is PSE meat, which is characterized by light colour, soft texture and a reduced water-binding capacity. The aim of the study was to compare plasma corticosterone concentration, muscle fibre diameter, and the apoptotic markers between normal and pale, soft, exudative (PSE) turkey breast muscles. The results show, that the PSE group is characterized by a significantly higher plasma corticosterone concentration and a significantly greater muscle fibre diameter at $30 \mathrm{~min}$ post mortem compared to the normal group. The time of refrigerated storage $\left(4^{\circ} \mathrm{C}\right)$ of meat contributes significantly to reducing muscle fibre diameter in the PSE group at $24 \mathrm{~h}$ post mortem compared to $30 \mathrm{~min}$ post mortem. Additionally, the results obtain showed that the caspase- 3 activity at 30 min post mortem increased significantly in the turkey breast muscles of PSE compared with the normal group. Moreover, the PSE group demonstrated the higher apoptotic potential measured in the breast muscle of turkey compared with the normal group. It is stated from the obtained results that the incidence of PSE meat in turkeys may be associated with their higher susceptibility to stress factors and stressinduced pathological states.

Keywords: corticosterone, muscle fibres, capase-3, apoptosis, PSE turkey meat

The growing demand for poultry meat and the economic considerations related to the short production cycle have led to the intensification of livestock production (3). One of the most rapidly developing fields of domestic animal production are turkeys (17), in which the intensive genetic selection over the last decade has markedly improved reproductive capacity, growth rate, and dressing percentage (1). However, the continuing progress in improving body weight and muscle weight in turkeys has contributed to metabolic and physiological disorders in muscle tissue structure and has had an adverse impact on the immunity and health of the birds (23).

The greatest concern in the poultry industry is PSE (pale, soft, exudative) meat, which is characterized by light colour, soft texture and reduced water-binding

The study was conducted as part of the project DS-3253/ZAZ and supported by a targeted research subsidy from the Ministry of Science and Higher Education in Poland. capacity (22). The low water-holding capacity of such meat limits its processing suitability, while the unnaturally light colour and soft texture make it less valuable as culinary meat (34), which results in enormous economic losses, lower yield, and reduced quality of the meat raw material $(3,4)$. The development of PSE meat is caused by acute stress experienced by animals immediately prior to slaughter (34). This causes a rapid breakdown of muscle glycogen, and the large amounts of the resulting lactic acid lead to muscle acidification (i.e. low $\mathrm{pH} 24 \mathrm{~h}$ post mortem), protein denaturation, and reduced waterholding capacity (8).

The frequency of PSE meat is related to stress-inducing factors, which have an impact on blood biochemical characteristics (28). The literature documents many physiological parameters indicative of stress in poultry. According to Puvadolpirod and Thaxton (27), one of the major indicators of stress in birds is the concentration 
of corticosterone, the main stress hormone, the blood concentration of which is 100 times as high as that of cortisol (14).

Stress response is tightly linked to meat quality such as colour, drip loss, or $\mathrm{pH}$ (32). Previous studies have shown that the first step of the conversion of muscle to meat is the apoptotic cell death (11). Apoptosis or programmed cell death is a highly conserved biological process that plays an important role in multicellular organisms, both during development, where it ensures the elimination of superfluous tissues, and in adulthood, where it is critical for maintenance of tissue homeostasis. In the case of skeletal muscle tissue, immediately after slaughter, after bleeding all skeletal muscle fibres will be deprived of oxygen and nutrients and rapidly undergo the apoptosis programme. Programmed cell death is triggered by external (death receptor pathway) or internal stimuli (mitochondrial pathway). In the intrinsic pathway, the cells undergo apoptosis because of cell stress, while the extrinsic pathway is induced by signals from other cells. Both pathways initiate apoptosis by activating caspases. However, Huang et al. (12) suggested that the intrinsic pathway, involving caspase- 3 activation and cytochrome c release from mitochondria to the cytoplasm, is the main apoptosis pathway during the conversion of muscle into meat. Moreover, earlier studies have also demonstrated the significance of PSE syndrome in programmed cell death (9). Therefore the expectations of producers and consumers as to explaining the increasing incidence of meat defects, which affect its quality, entail performing detailed studies of muscle tissue structure. The aim of the study was to compare plasma corticosterone concentration and muscle fibre diameter, the post mortem apoptotic markers between normal and PSE turkey breast muscles.

\section{Material and methods}

From 238 turkey hens (BUT-9 line) derived from one flock, a total of 54 boneless skinless whole turkey breast muscles were collected from the deboning lines of a local commercial processing plant 'Eco-drób' in Lubomierz. The birds were 16 weeks old and their live weight was $9.5 \mathrm{~kg}$. The $\mathrm{L}^{*}$ values were measured with a colorimeter on a total of 238 breast muscles on the deboning line to determine one of two colour categories according to Owens et al. (22) criteria; namely normal group $(\mathrm{n}=30)$ having $\mathrm{L}^{*}$ values lower than 53 and PSE group $(n=24)$ having $L^{*}$ values greater than 53. Colour was measured on the cranial, medial surface (bone side) in an area free of obvious colour defects.

To determine corticosterone concentration, immediately after slaughter the blood samples were collected from each bird in heparinised tubes containing sodium fluoride and stored $1 \mathrm{~h}$ at $4^{\circ} \mathrm{C}$, after which they were centrifuged at 4,000 rpm for $20 \mathrm{~min}$ to remove blood cells and obtain plasma. Individual plasma samples from each turkey were kept at $-20^{\circ} \mathrm{C}$ until analysis. Plasma corticosterone levels were measured by enzyme-immunoassay using a commercial kit (Diagnostic Systems Laboratories, USA) according to the manufacturer's instructions. All samples were run in duplicate and kit calibrators and controls were included in each analysis. Absorbance was measured at $450 \mathrm{~nm}$, with a reference wave length of $650 \mathrm{~nm}$, in an ELISA Microplate Reader (Sirio-S, Canada).

Moreover, to determine muscle fibre size, the experiment used breast muscle fragments of turkey hens, collected from the left carcass side $30 \mathrm{~min}$ post mortem, and after 24, 48 and $72 \mathrm{~h}$ of refrigerated storage $\left(4^{\circ} \mathrm{C}\right)$. Tissue samples were frozen in isopentane cooled with liquid nitrogen and stored at $-80^{\circ} \mathrm{C}$ until analysis. Material prepared in this way was mounted on specimen holders using frozen tissue medium, and then cut on a cryostat (Slee MEV, Germany) at $-20^{\circ} \mathrm{C}$ into $10 \mu \mathrm{m}$-thick sections. Frozen sections were fixed on Super-Frost glass slides treated with Poly-L-lysine solution. To contrast breast muscle sections, cryopreparations were stained with hematoxylin and eosin, and after dehydrating in a graded ethyl alcohol series, they were cleared in xylene and embedded in Canada balm.

For the evaluation of apoptotic nuclei, the cryopreparations from 30 min post mortem were used and apoptotic nuclei were detected using the ApopTag Plus Peroxidase In Situ Apoptosis Detection Kit (Chemicon International, Melbourne, Australia) following the manufacturer's protocol. In brief, frozen muscle sections were fixed in 1\% paraformaldehyde for $10 \mathrm{~min}$ at RT, post-fixed in precooled ethanol: acetic acid $2: 1$ for $5 \mathrm{~min}$ at $-20^{\circ} \mathrm{C}$, and blocked in $3 \% \mathrm{H}_{2} \mathrm{O}_{2}$ for $5 \mathrm{~min}$ at RT to quench endogenous peroxidase activity. Samples were incubated in an equilibration buffer for $10 \mathrm{~min}$ at RT in a humidified chamber, after which working strength TdT enzyme was added to sections, which were incubated in a humidified chamber at $37^{\circ} \mathrm{C}$ for $1 \mathrm{~h}$. The slides were then rinsed with wash buffer for $10 \mathrm{~min}$ at RT followed by a $5 \mathrm{~min}$ PBS (phosphate-buffered saline) wash. Excess of liquid was tapped off and the anti-digoxigenin conjugate was applied directly to the specimens and incubated for $30 \mathrm{~min}$ at RT in a humidified chamber. After rinsing in PBS, apoptotic cells were visualized by the addition of DAB solution. A negative control was performed without active TdT enzyme to control for non-specific incorporation of nucleotides or for nonspecific binding of enzyme conjugate. All the sections were dehydrated through an increased series of ethanol and mounted under glass with DPX.

The enzymatic activity of caspase- 3 in the breast muscle samples of turkey hens, collected from the left carcass side 30 min post mortem, was determined in a tissue homogenate using the ready-to-use Fluorometric Assay Kit (K105-100, Bio Vision, Inc., Milpitas, Calif., USA) according to the manufacturer's instructions. Briefly, tissue samples were homogenized in a lysis buffer (100 mg tissue per $300 \mu \mathrm{l}$ buffer) and centrifuged $\left(12000 \times \mathrm{g}, 20 \mathrm{~min}, 4^{\circ} \mathrm{C}\right)$. Supernatants were collected and total protein concentrations were determined by the bicinchoninic acid (BCA) protein assay. Next, a $100 \mu \mathrm{g}$ aliquot of protein from each sample or blank control were transferred in duplicate to 96 -well microplates. Immediately after a $2 \mathrm{~h}$ incubation at $37^{\circ} \mathrm{C}$ in a reaction buffer with substrate, fluorescence at $400 \mathrm{~nm}$ excitation and $505 \mathrm{~nm}$ emission was read in a Fluorescence Microplate Reader FLx800 (BioTek Instruments, Winooski, Vt., USA). The activity of caspase-3 in the samples of PSE breast muscle group was normalized and expressed as the percentage activity relative to the normal breast muscle group (see at $100 \%$ ).

Microscopic preparations were analysed under a Nikon E600 light microscope (Japan), and the measurements were performed using computer-based image analysis system 
MultiScan v. 14.02 (Poland). Muscle fibre diameters were estimated based on 100 randomly chosen fibres in each preparation and in all meat storage periods. However, the TUNEL-positive nuclei were counted on at least 10 different fields $\left(500 \mu \mathrm{m}^{2}\right)$ of each sample and the number of positive nuclei was expressed per unit area.

The results were statistically analysed based on SAS 9.2 Academic Analysis Suite (2011). Least square means (LSM) and standard errors (SE) were determined for all of the analysed parameters. The calculations were made using the twoway analysis of variance. The main effects were the analysed bird groups and the meat storage time. Significant differences between the means were analysed with the Tukey test. The 0.05 significance was accepted to represent significant differences between means.

\section{Results and discussion}

The plasma level of stress hormones plays an important role in evaluating the sensitivity of animals to environmental factors. The study, which investigated corticosterone concentration in two turkey groups, showed that the PSE group has a significantly higher plasma concentration of corticosterone, one of the major hormones involved in the body's response to stress, compared to the normal group (Tab. 1). The obtained results confirm the earlier study of Kannan et al. (15), who analysed the effect of elevated plasma corticosterone concentration on meat quality characteristics of breast muscles in broiler chickens and noted that artificially elevating circulating plasma corticosterone concentration is associated with lighter coloured muscles. Based on the presented results the authors assume that the high plasma corticosterone concentration may be related to the presence of PSE meat in poultry. This view is shared by Yoshioka et al. (36), who investigated the effect of cortisol on muscle proteolysis and meat quality of the longissimus muscle in piglets, and showed that high concentrations of cortisol, which is an equally reliable stress hormone in mammals, added to feed for 7 days, stimulates the activity of calpains ( $\mu$-calpain and m-calpain) and accelerates muscle proteolysis, which may contribute to PSE meat. Martinez-Rodriguez et al. (20) also demonstrated that high plasma cortisol concentrations are caused by

Tab. 1. Comparison of corticosterone concentration, muscle fibre diameter, caspase-3 activity and number of apoptotic nuclei between normal and PSE groups of turkeys $(\mathrm{LSM} \pm \mathrm{SE})$

\begin{tabular}{|l|c|c|}
\hline \multicolumn{1}{|c|}{ Traits } & Normal group & PSE group \\
\hline Corticosterone concentration $(\mathrm{ng} / \mathrm{ml})$ & $4.12 \pm 0.15^{\mathrm{a}}$ & $9.76 \pm 0.38^{\mathrm{b}}$ \\
\hline $\begin{array}{l}\text { Muscle fibre diameters }(\mu \mathrm{m}) \\
30 \mathrm{~min}\end{array}$ & $54.38 \pm 0.08^{\mathrm{ax}}$ & $57.25 \pm 0.76^{\mathrm{bx}}$ \\
$24 \mathrm{~h}$ & $53.97 \pm 0.06^{\mathrm{ax}}$ & $54.69 \pm 0.52^{\mathrm{ay}}$ \\
$48 \mathrm{~h}$ & $53.52 \pm 0.59^{\mathrm{ax}}$ & $54.16 \pm 0.65^{\mathrm{ay}}$ \\
$72 \mathrm{~h}$ & $53.81 \pm 0.73^{\mathrm{ax}}$ & $54.53 \pm 0.77^{\mathrm{ay}}$ \\
Caspase-3 activity (\%) & $100 \pm 1.08^{\mathrm{a}}$ & $148.50 \pm 1.67^{\mathrm{b}}$ \\
Number of apoptotic nuclei & $8.56 \pm 0.03^{\mathrm{a}}$ & $12.24 \pm 0.04^{\mathrm{b}}$ \\
\hline
\end{tabular}

Explanations: $a, b$ - means with a different letter differ significantly between normal and PSE groups of turkeys at $\mathrm{P} \leq 0.05$; $\mathrm{x}, \mathrm{y}-$ means with a different letter differ significantly between periods of refrigerated meat storage at $\mathrm{P} \leq 0.05$ prolonged exposure to stress-inducing factors, which leads to incidence of meat defects, including PSE meat, in pigs. A similar view is held by Hambrecht et al. (10), who studied the effects of transportation, lairage, and preslaughter stressor treatment on the glycolytic potential and pork quality of the glycolytic longissimus muscle and the oxidative supraspinatus or serratus ventralis muscles, and found that in the glycolytic muscle the high physical and psychological stress levels associated with stress in the immediate preslaughter period have a greater effect on the water-holding capacity of the meat and may promote PSE development. In turn, Shaw and Trout (30), when analysing cortisol concentrations in longissimus muscle from PSE pork, showed that both plasma and muscle cortisol concentration of PSE carcasses did not differ significantly from normal carcass, and PSE meat is regarded as a 'stress-related' condition. Meanwhile, Kowalska et al. (16), who determined stress levels in the blood of two rabbit groups (unstressed and passive), noted significantly higher cortisol concentrations in passive rabbits with PSE meat compared to the group of unstressed rabbits. According to the authors, this is related to the adaptive processes and may result from a rapid decline in $\mathrm{pH}$ value and from the abnormal glycolysis and maturation of meat which are characteristic of PSE meat.

Poznyakovskiy et al. (25) consider that one of the main causes of PSE meat is susceptibility of poultry to genetically determined stress factors. Therefore, the differences shown in the study in corticosterone concentration in the analysed turkey groups are probably due to the different sensitivities of birds to stress. Significantly higher plasma corticosterone concentration for the PSE group compared to the normal group may be attributed to the greater susceptibility of turkeys to stress factors and stress-induced pathological states. A similar opinion is expressed by Kowalska et al. (16), who demonstrated that PSE meat is more frequent in stress-susceptible animals, although the concentration of cortisol is also largely dependent on the bird's individual reaction to stress factors before slaughter.

Muscle fibre diameter is one of the factors influencing many biochemical processes in meat, which, in turn, determine its ultimate quality. The study, which analysed two groups of turkeys, i.e. normal and PSE groups, showed that the PSE group is characterized by a significantly greater fibre diameter at 30 min post mortem compared to the normal group (Tab. 1). In the successive periods of refrigerated storage of meat $(24,48$ and $72 \mathrm{~h}$ post mortem) there were no significant differences in muscle fibre diameter between the analysed turkey groups. These results corroborate the earlier findings of Lesiow et al. (19), who analysed the effect of the autumn-winter period on the ultrastructure of PSE and normal meat in chickens and showed significantly higher fibre diameter of the pectoralis major muscle for PSE meat compared to normal meat. Likewise, Wojtysiak and Migdał (33), who investigated differences in the size of muscle fibres between normal and malignant hyperthermia susceptibility (MHS) pigs, which is strictly associated with PSE pork meat (26), demonstrated that the longissimus muscle of the heterozygous $\mathrm{Nn}$ fatteners had a significantly larger 
diameter of all the examined muscle fibre types compared to muscles from the homozygous NN group. This view is supported by Fiedler et al. (7), who analysed structural and functional characteristics of longissimus muscle fibres in pigs with different MHS and showed that pigs which were heterozygous Nn fatteners for the MHS had a significantly larger diameter of all examined muscle fibre types compared to the muscle from homozygous NN group. In turn, Lengerken et al. (18), who compared the metabolism of longissimus muscle with PSE and normal meat in pigs, noted that mean fibre diameter of all types was significantly increased in pigs developing PSE meat after slaughter, compared to pigs with normal meat quality.

As regards the effect of the time of refrigerated storage of meat on muscle fibre diameter, the analysis of the results presented in the study showed that refrigerated meat storage time has a significant effect on reducing muscle fibre diameter at $24 \mathrm{~h}$ post mortem compared to $30 \mathrm{~min}$ post mortem, but only for the PSE group of turkeys (Tab. 1). In subsequent periods of refrigerated meat storage, i.e. at 48 and $72 \mathrm{~h}$ post mortem, no significant differences were observed in muscle fibre diameter in both turkey groups analysed. Diesbourg et al. (5) hold the view that the decrease in muscle fibre size during storage of meat is due to shrinkage of lateral connections between myofibrils and sarcolemma. In turn, Huff-Lonergan and Lonergan (13) indicate that muscle fibre size post mortem is determined by the level of drip loss and by the degradation rate of cytoskeletal proteins post mortem, in particular the rate of desmin degradation. This protein connects myofibrils not only with each other, but also with the cell membrane, thus contributing to the formation of intracellular protein complexes known as costameres. As reported by Carvalho et al. (3), Chan et al. (4) and Owens et al. (22) PSE meat of turkeys is characterized by high drip loss. In turn, pig research showed that PSE meat is characterized by slower desmin degradation post mortem compared to RFN meat (35). Therefore, the reduction in muscle fibre size, observed in the study 30 min post mortem, can be related in the case of the PSE group to both the high drip loss and the relative slow rate of desmin degradation, since according to the hypothesis of Huff-Lonergan and Lonergan (13) muscle fibres in which desmin degrades slowly post mortem, reduce their diameter even when drip loss is low.

The stress to which animals are subjected before slaughter increases the concentration of calcium ions in the sarcoplasm and causes a rapid breakdown of muscle glycogen, while the lactic acid generated in large quantities acidifies the muscles (28) as well as causing numerous functional and morphological modifications of muscle fibres, which ultimately lead to their death, mainly through apoptosis (2). Increased calcium ion concentration, oxidative stress, as well as elevated level of reactive oxygen species (ROS), electrolyte transport abnormalities, and DNA damage, are the main factors inducing apoptosis, or programmed cell death. Apoptosis is coordinated by a family of cystein proteases, the caspases that dismantle the cell by targeting a panoply of proteins for limited proteolysis. The caspase plays a central role in signal transduction and final execution in apoptotic processes (29). According to the homology of their big and small subunits and their functions in apoptosis, caspases involved in apoptosis can be classified into two types: the initiator caspases, which include caspase-2, -8, -9 and -10; and the effector caspases, which include caspase- $3,-6$, and -7 . In the current research the activity of caspase- 3 measured $30 \mathrm{~min}$ post mortem in the turkey breast muscle of PSE and normal group is presented in Tab. 1. The results obtained showed that compared with the normal group, the caspase-3 activity increased significantly in the PSE group. These results support an earlier study by Guo et al. (9) in which the authors analysed the protein expression levels and enzyme activities of the functional apoptotic factors to determine the differences in apoptosis status between porcine PSE and RFN pork meat and found significantly higher activity of caspase-3 in PSE meat $1 \mathrm{~h}$ post mortem compared to RFN meat. Moreover, the authors showed that the higher potential of increased sarcoplasmic calcium concentrations presented in PSE meat may induce apoptosis. Higher apoptotic potential in the PSE group is also confirmed by the microscopic examinations performed in the present study (Fig. 1,

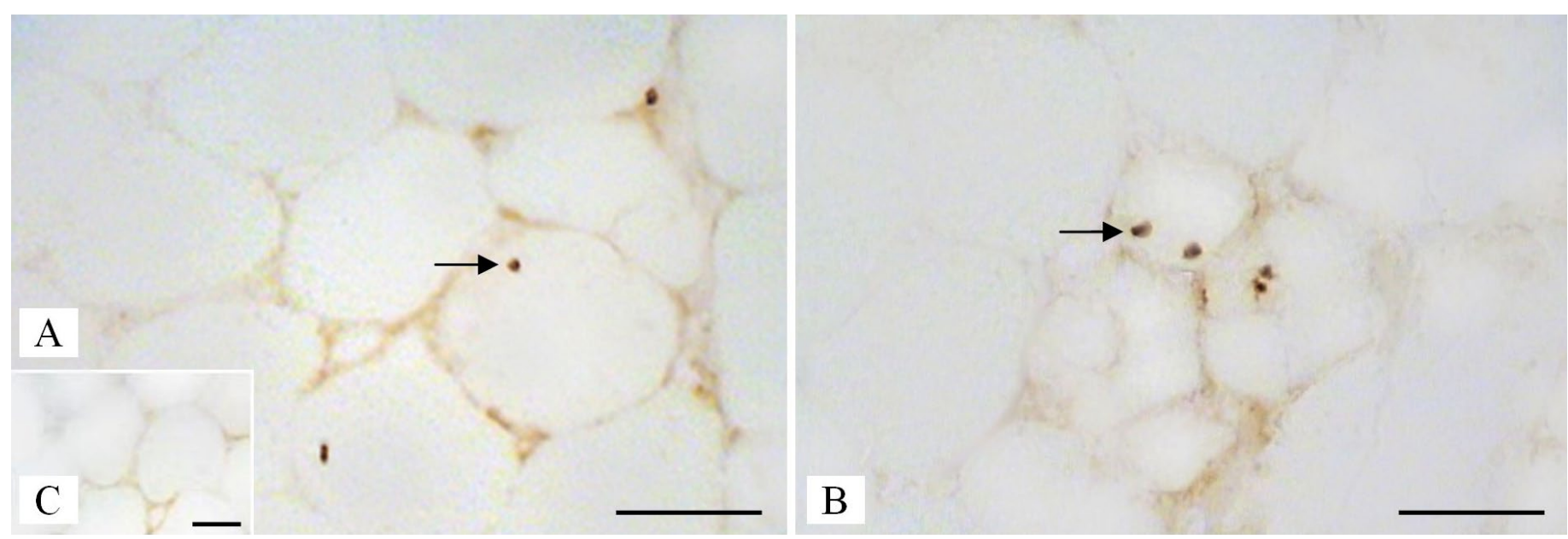

Fig. 1. Localization of apoptotic nuclei in the breast muscle from normal (A) and PSE (B) groups of turkeys

Explanations: Apoptotic nuclei were identified using TUNEL method. Arrows indicate single apoptotic nuclei. A negative control for TUNEL assay was performed without active TdT enzyme (C inset). Scale bar $50 \mu \mathrm{m}$ 
Tab. 1). We showed that apoptosis as measured by TUNEL staining was increased in breast muscles of the PSE group compared with the normal group. There was no staining detection in a negative control where TdT enzyme was omitted (Fig. 1C inset). However, it is worth noting that in both the PSE and normal group, apoptosis involved only single nuclei in the muscle fibres, which indicates that nuclei within one continuous cytoplasmic area can respond differently to similar signals. These results support the earlier idea that the fate of nuclei can be different; that nuclei have some autonomy. Dipasquale and Youle (6) and Polunovsky et al. (24) indicated that apoptosis can occur in one or more nuclei within the same cytoplasm without induction of apoptosis in the other nuclei. Newlands et al. (21) showed that within an individual muscle fibre, not all nuclei are transcriptionally equivalent. Likewise Siu et al. (31) confirmed that nuclei which reside in the muscle fibres in close proximity to each other are capable of responding to similar cytoplasmic signals in a distinct manner. Exactly how this nuclear independency is regulated is currently unknown. In most cell types the morphological characteristics of apoptosis are consistent. However, apoptosis in skeletal muscles has been relatively unexplored. Skeletal muscle is unique in that it is multi-nucleated, and evidence suggests that individual decay of muscle fibre nuclei is a more frequent occurrence than wholesale muscle fibre cell death.

In summary, obtained results show that the PSE syndrome contributes an greater muscle fibre diameter at 30 min postmortem and increase in corticosterone level, which suggests a higher sensitivity of animals to stress factors. In addition, the significantly increased caspase-3 activity and the higher number of apoptotic nuclei suggested higher apoptotic potential in PSE group compared with normal group of turkey.

\section{References}

1. Budnik S., Burek R.: Turkeys production - chances and threats. XI Symp. of Poultry PTNW, Wrocław, Polska, czerwiec 2009, p. 35-40 (in Polish).

2. Cao J., Sun W., Zhou G., Xu X., Peng Z., Hu Z.: Morphological and biochemical assessment of apoptosis in different skeletal muscles of bulls during conditioning. J. Anim. Sci. 2010, 88, 3439-3444.

3. Carvalho R. H., Soares A. L., Honorato D. C. B., Guarnieri P. D., Pedrão M. R., Paião F. G., Oba A., Ida E. I., Shimokomaki M.: The incidence of pale, soft, and exudative (PSE) turkey meat at a Brazilian commercial plant and the functional properties in its meat product. LWT - Food Sci. Technol. 2014, 59, 883-888.

4. Chan J. T. Y., Omana D. A., Betti M.: Application of high pressure processing to improve the functional properties of pale, soft, and exudative (PSE)-like turkey meat. Innov. Food Sci. Emerg. Technol. 2011, 12, 216-225.

5. Diesbourg L., Swatland H. J., Millman B. M.: X-ray diffraction measurements of post mortem changes in the myofilament lattice of pork. J. Anim. Sci. 1988, $66,1048-1054$

6. Dipasquale B., Youle R. J.: Programmed cell death in heterokaryons. A study of the transfer of apoptosis between nuclei. Am. J. Pathol. 1992, 141, 1471-1479.

7. Fiedler I., Ender K., Wicke M., MaakS, von Lengerken G., Meyer W.: Structural and functional characteristics of muscle fibres in pigs with different malignant hyperthermia susceptibility (MHS) and different meat quality. Meat Sci. 1999, $53,9-15$

8. Fraqueza M. J., Cardoso A. S., Ferreira M. C., Barreto A. S.: Incidence of pectoralis major turkey muscles with light and dark color in a Portuguese slaughterhouse. Poult. Sci. 2006, 85, 1992-2000.

9. Guo B., Zhang W., Tume R. K., Hudson N. J., Huang F., Yin Y., Zhou G.: Disorder of endoplasmic reticulum calcium channel components is associated with the increased apoptotic potential in pale, soft, exudative pork. Meat Sci. 2016, 115, $34-40$.

10. Hambrecht E., Eissen J. J., Newman D. J., Smits C. H. M., Verstegen M. W. A., den Hartog L. A.: Preslaughter handling effects on pork quality and glycolytic potential in two muscles differing in fiber type composition. J. Anim. Sci. 2005, 83, 900-907.
11. Herrera-Mendez C. H., Becila S., Boudjellal A., Ouali A.: Meat ageing: Reconsideration of the current concept. Trends Food Sci. Technol. 2006, 17, 394-405.

12. Huang F., Huang M., Zhang H., Zhang C., Zhang D., Zhou G.: Changes in apoptotic factors and caspase activation pathways during the post mortem aging of beef muscle. Food Chem. 2016, 190, 110-114.

13. Huff-Lonergan E., Lonergan S. M.: Mechanisms of water-holding capacity of meat: The role of post mortem biochemical and structural changes. Meat Sci. 2005, 71, 194-204.

14. Jong I. C. de, van Voorst A. S., Erkens J. H., Ehlhardt D. A., Blokhuis H. J.: Determination of the circadian rhythm in plasma corticosterone and catecholamine concentrations in growing broiler breeders using intravenous cannulation. Physiol. Behav. 2001, 74, 299-304.

15. Kannan G., Heath J. L., Wabeck C. J., Owens S. L., Mench J. A.: Elevated plasma corticosterone concentrations influence the onset of rigor mortis and meat color in broilers. Poult. Sci. 1998, 77, 322-328.

16. Kowalska D., Gugotek A., Bielański P.: Effect of stress on rabbit meat quality. Ann. Anim. Sci. 2011, 11, 465-475.

17. Koziot I., Krzywon M.: The condition of the poultry industry in Poland. Prog. Econom. Sci. 2014, 1, 85-98 (in Polish).

18. Lengerken G. von, Maak S., Wicke M.: Muscle metabolism and meat quality of pigs and poultry. Vet. Zootech-Lith. 2002, 20, 82-86.

19. Lesiów T., Szmanko T., Korzeniowska M., Bobak Ł., Oziembłowski M.: Influence of the season of the year on some technological parameters and ultrastructure of PSE, normal and DFD chicken breast muscles. $19^{\text {th }}$ Europ. Symp. Quality Poultry Meat, 21-25 June 2009, Turku, Finland, p. 1-8.

20. Martinez-Rodriguez R., Roldan-Santiago P., Flores-Peinado S., Ramirez-Telles J. A., Mora-Medina P., Trujillo-Ortega M. E., Gonzalez-Lozano M., BecerrilHerrera M., Sanchez-Hernandez M., Mota-Rojas D.: Deterioration of pork quality due to the effects of acute ante mortem stress: An overview. Asian J. Anim. Vet. Adv. 2011, 6, 1170-1184.

21. Newlands S., Levitt L. K., Robinson C. S., Karpf A. B., Hodgson V. R., Wade R. P., Hardeman E. C.: Transcription occurs in pulses in muscle fibers. Genes Dev. 1998, 12, 2748-2758.

22. Owens C. M., Hirschler E. M., McKee S. R., Martinez-Dawson R., Sams A. R. The characterization and incidence of pale, soft, exudative turkey meat in a commercial plant. Poult. Sci. 2000, 79, 553-558.

23. Petracci M., Mudalal S., Soglia F., Cavani C.: Meat quality in fast-growing broiler chickens. World's Poult. Sci. J. 2015, 71, 363-374

24. Polunovsky V. A., Ingbar D. H., Peterson M., Bitterman P. B.: Cell fusion to study nuclear-cytoplasmic interactions in endothelial cell apoptosis. Am. J. Pathol. 1996, 149, 115-128.

25. Poznyakovskiy V. M., Gorlov I. F., Tikhonov S. L., Shelepov V. G.: About the quality of meat with PSE and DFD properties. Foods and Raw Mater. 2015, 3, 104-110

26. Pribula M., Turek P., Kováč G.: Effect of PSE syndrome on the pork quality. XV Kongres Polskiego Towarzystwa Nauk Weterynaryjnych, 22-24 September 2016, Lublin, Poland, p. 223

27. Puvadolpirod S., Thaxton J. P.: Model of physiological stress in chickens 2. Dosimetry of adrenocorticotropin. Poult. Sci. 2000, 79, 370-376.

28. Qu D., Zhou X., Yang F., Tian S., Zhang X., Ma L., Han J.: Development of class model based on blood biochemical parameters as a diagnostic tool of PSE meat. Meat Sci. 2017, 128, 24-29.

29. Rupinder S. K., Gurpreet A. K., Manjeet S.: Cell suicide and caspases. Vascular Pharmacol. 2007, 46, 383-393.

30. Shaw F. D., Trout G. R., McPhee C. P.: Plasma and muscle cortisol measurements as indicators of meat quality and stress in pigs. Meat Sci. 1995, 39, 237-246.

31. Siu P. M., Pistilli E. E., Butler D. C., Alway S. E.: Aging influences cellular and molecular responses of apoptosis to skeletal muscle unloading. Am. J. Physiol. Cell Physiol. 2005, 288, C338-C349.

32. Śmiecińska K., Denaburski J., Sobotka W.: Slaughter value, meat quality, creatine kinase activity and cortisol levels in the blood serum of growing-finishing pigs slaughtered immediately after transport and after a rest period. Pol. J. Vet. Sci. 2011, 14, 47-54.

33. Wojtysiak D., Migdat W.: Differences of muscle fibre composition and tenderness of $\mathrm{m}$. longissimus lumborum between heterozygous and homozygous negative Polish Landrace pigs for the RYR1. Arch. Tierz., Dummerstorf 2007, 50, 186-193.

34. Wójcik A., Pomianowski J. F., Sowińska J., Mituniewicz T., Witkowska D., Choraży E., Piotrowska J.: Effect of pre-slaughter handling of broiler chickens on meat technological quality. Inż. Ap. Chem. 2011, 50, 85-86 (in Polish).

35. Yin Y., Zhang W. G., Zhou G. H., Guo B.: Comparison of protein degradation, protein oxidation, and $\mu$-calpain activation between pale, soft, and exudative and red, firm, and nonexudative pork during post mortem aging. J. Anim. Sci. 2014, 92, 3745-3752.

36. Yoshioka G., Imaeda N., Ohtani T., Hayashi K.: Effects of cortisol on muscle proteolysis and meat quality in piglets. Meat Sci. 2005, 71, 590-593.

Corresponding author: Dorota Wojtysiak, PhD, Al. Mickiewicza 24/28, 30-059 Kraków, Poland; e-mail: wojtysiakd@wp.pl 